\title{
DESENVOLVIMENTO DE ÍNDICE DE SELEÇÃO EM GADO CORTE SOB O ENFOQUE DE UM MODELO BIOECONÔMICO*
}

\section{DEVELOPMENT OF SELECTION INDEX IN BEEF CATTLE UNDER THE FOCUS OF A BIO-ECONOMICMODEL}

\author{
Brumatti, R.C. ${ }^{1}$, Ferraz, J.B.S. ${ }^{2}$, Eler, J.P. ${ }^{2}$ e Formigonni, I.B. ${ }^{2}$
}

${ }^{1}$ Departamento de Zootecnia. FAMEZ. UFMS. Av. Senador Filinto Muller, $n^{\circ} 2443$. Caixa Postal 549. CEP 79070-900. Campo Grande-MS. Brasil.rbrumatti@gmail.com ${ }^{2}$ Departamento de Ciências Básicas. GMAB. FZEA. USP. Brasil.

\section{PaLAVRAS ChaVE ADICIONAIS}

Melhoramento animal. Objetivo de seleção. Ponderador econômico.

\section{RESUMO}

O presente trabalho teve por objetivo desenvolver um modelo bioeconômico para determinação de ponderadores econômicos utilizados em índices de seleção em programas de melhoramento genético de bovinos de corte. Fo desenvolvido um cenário padrão, onde, em termos absolutos, as características de maior relevância econômica foram o rendimento de carcaça, as características ligadas à fertilidade do rebanho, seguidas pelas características de peso e ganho de peso e por último as referentes à mortalidade. Os valores genético-econômicos obtidos foram, para habilidade de permanência (HP) 0,879 ; para probabilidade de prenhes ao 14 meses de idade (PP14) 0,201; para ganho de peso pós-desmama (GPD245) 0,190; para peso a desmama (PD) 0,061; e 0,080 para peso ao sobreano (PS). Portanto, verificou-se que as características reprodutivas, HP e PP14, foram de 4,28 a 13,46 vezes mais importantes economicamente do que as características de crescimento GPD245, PD e PS.

\section{SUMMARY}

Aiming to increase the economical studies applied to animal genetic improvement, the present work had as goal to develop a bio-economical model to determine the economical weights utilized on indexes of selection in genetic improvement

*Pesquisa financiada pela FAPESP, CNPq e CAPES.

Recibido: 2-1-09. Aceptado: 22-7-09.

\section{AdDitIONAL KEYWORDS}

Breeding goals. Selection objective. Economic values.

programs in beef cattle. A standard scenario was developed where, in absolute terms, the traits with major economical relevance were respectively, carcass yield, traits related to herd fertility, followed by those related to weight and weight gain, and last, referred to mortality. The geneticeconomical values obtained were 0.897 for stayability, 0.201 for heifer pregnancy at 14 months (PP14), 0.190 for pos-weaning weight $/ \mathrm{kg} /$ day, 0.061 for weaning weight and 0.080 for steer weight at $18 \mathrm{mo}$. Therefore, it was verified that the reproductive traits, stayability and PP14, were 4.28 and 13.46 times economically more important than the growth traits, pos-weaning weight, weaning weight and steer weight at 18 months.

\section{INTRODUÇÃO}

Toda atividade produtiva deve ter por premissa básica a busca por resultados eficientes em termos produtivos e, sobretudo em termos econômicos.

Nesse sentido, a gestão da propriedade rural tem apresentado relevante importância, sendo que o aumento da eficiência da pecuária de corte no Brasil, passa necessariamente pela melhoria da qualidade genética dos rebanhos, que pode ser obtida, principalmente, pela escolha dos indivíduos que serão os pais da geração seguinte e 


\section{BRUMATTI, FERRAZ, ELERE FORMIGONNI}

direcionamento dos acasalamentos (Lôbo et al., 1999).

Contudo, a maior dificuldade na identificação de tais indivíduos consiste no fato de que o mérito genético, representado pelo conjunto de genes ou seus genótipos, não é mensurável diretamente, e deve ser predito, sendo objetivo central da avaliação genética.

O uso de avaliações genéticas possibilita de maneira mais acurada, a escolha de animais para a formação do patrimônio genético, corroborando para a resposta ao questionamento feito por Paneto (1998), "... entre os animais disponíveis no presente, quais devem ser escolhidos para formar o patrimônio genético do futuro?"

Contudo, segundo Bourdon and Golden (2000), a decisão de seleção dos animais melhoradores, envolve tanto questões genéticas como econômicas. Além disso, uma vez que a ponderação econômica para cada característica esteja correta, a pergunta "Qual o retorno esperado, em lucro, para unidade de mudança genética de uma característica?", já bastante questionada entre os pecuaristas, poderá ser mais facilmente respondida.

A união de avaliações genéticas e econômicas complementa o estudo do melhoramento genético animal, com a determinação das características a serem selecionadas e sua importância real dentro do sistema de produção. Isto vem de encontro com Ponzoni et al. (1989), que citaram que estudos de seleção genética são divididos em duas grandes áreas, determinação de valores genéticos e estimação de valores econômicos de características.

Com isso, a definição dos valores econômicos das características componentes dos índices de seleção trará uma contribuição importantíssima para uma maior eficiência econômica dos processos de avaliação genética, visando aumentar a eficiência produtiva e econômica da pecuária de corte (Amer et al., 2001 e Jorge et al. 2007).
O presente estudo teve como objetivo a determinação dos ponderadores econômicos aplicáveis às avaliações genéticas das características produtivas e reprodutivas em bovinos de corte da raça Nelore com o uso de um modelo bioeconômico.

\section{MATERIALE MÉTODOS}

O estudo foi desenvolvido para as condições de criação em bovinos de corte encontradas no Brasil Central, em outras palavras, animais Nelores criados em pastagens e clima tropicais.

A caracterização da propriedade utilizada como padrão para esta simulação está descrita na tabela I, considerando que os preços de mercado utilizados são relativos a junho de 2008 e praticados na Praça de Campo Grande, MS.

Para desenvolver o modelo bioeconômico foram utilizadas planilhas eletrônicas, com o uso da interligação entre os índices zootécnicos, a estrutura de rebanho, e os centros de custos e receitas. Foi possível verificar e obter os principais índices de produtividade, índices econômicos, além

Tabela I. Caracterização técnica-econômica da propriedade padrão utilizada no modelo bioeconômico. (Characterization technicaleconomic of default farm used in the bio-economic model).

\begin{tabular}{lc}
\hline Parâmetros & Valores \\
\hline Tamanho da propriedade (ha) & 2500 \\
Quantidade de matrizes (cabeças) & 1000 \\
Fertilidade de vacas adultas \% & 80,0 \\
Descarte de matrizes \% & 20,0 \\
Peso a desmama $(\mathrm{kg})$ & 184,0 \\
Peso ao sobreano de machos $(\mathrm{kg})$ & 324,0 \\
Peso ao sobreano de fêmeas $(\mathrm{kg})$ & 272,0 \\
Preço do boi gordo $(\mathrm{R} \$ / \mathrm{kg})$ & 5,86 \\
Preço da vaca gorda $(\mathrm{R} \$ / \mathrm{kg})$ & 5,26 \\
Preço do bezerro $(\mathrm{R} \$ / \mathrm{kg}$ vivo) & 3,80 \\
\hline
\end{tabular}

US $\$ 1,00=R \$ 1,61$. 


\section{ÍNDICE DE SELEÇÃO EM GADO CORTE SOB O ENFOQUE BIOECONÔMICO}

de custos por categoria produzida e os lucros marginais envolvidos nas simulações.

As características analisadas foram: precocidade sexual, expressadas como a probabilidade da novilha Nelore ficar prenhe quando exposta aos touros aos 14 meses de vida(PP14), habilidade de permanência(HP) das matrizes do rebanho, peso a desmama (PD), peso ao sobreano (PS) e o ganho de peso pós-desmama (GPD245).

O modelo bioeconômico realiza uma seqüência de atividades para poder determinar o cenário produtivo utilizado como padrão nesta simulação, onde tais seqüências são descritas na figura 1 .

Uma vez definido o cenário padrão o modelo bioeconômico irá mensurar o efeito de cada alteração nas características avaliadas. Para tanto se tem a necessidade de se respeitar uma seqüência de atividades estabelecidas através de uma ferramenta computacional desenvolvida no próprio MS Excel ${ }^{\circledR}$, onde a seqüência de passos é demonstrada na figura 2 .

O valor econômico de uma dada característica é dado pela seguinte equação:

$V L=\Delta L / \Delta G$

onde:

VE = Valor econômico da característica;

$\Delta \mathrm{L}=$ Variação no lucro;

$\Delta \mathrm{G}=$ Variação no desempenho da característica em análise.

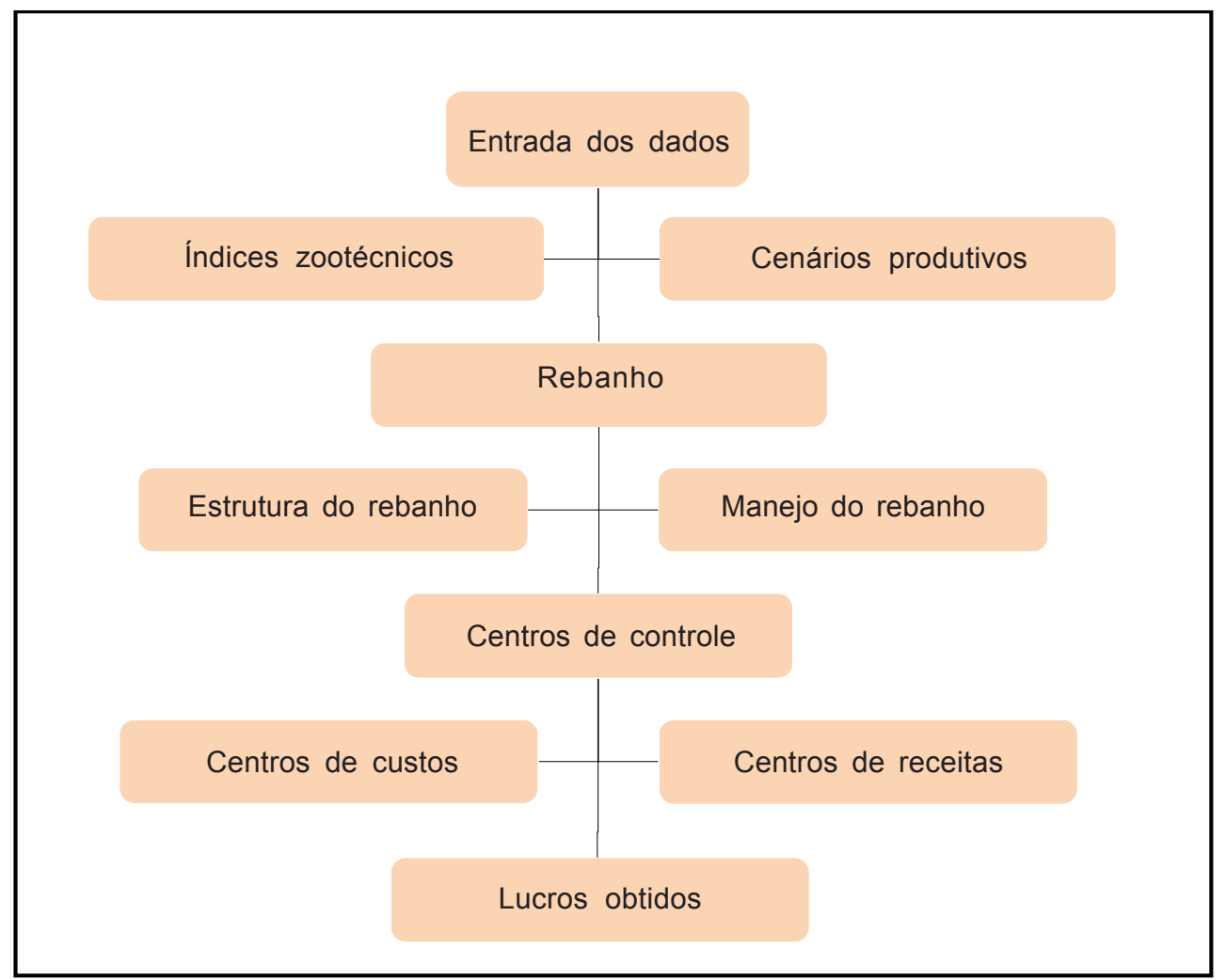

Figura 1. Organograma das atividades realizadas pelo modelo bioeconômico. (Organizational chart of the activities carried out by the bio-economic model). 

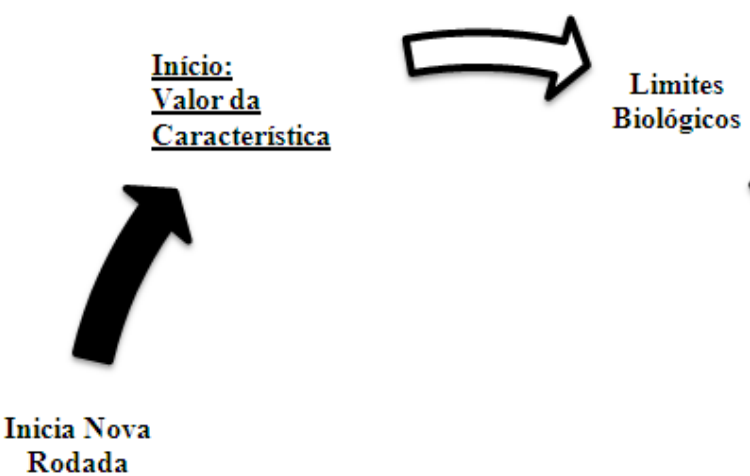

Rodada
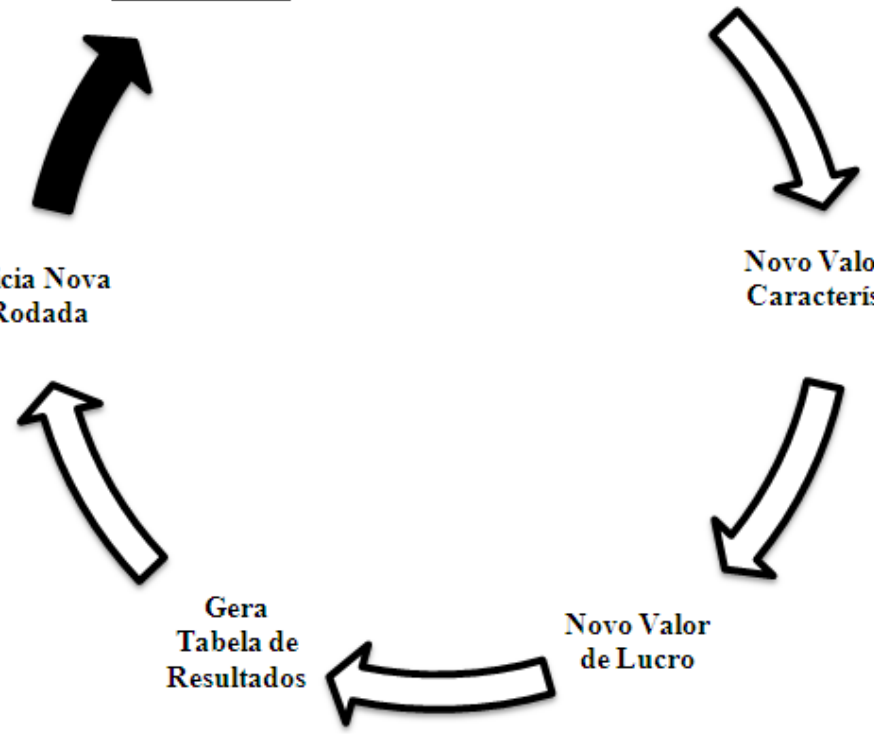

Novo Valor da

Característica

Figura 2. Seqüencia de atividades realizadas pelo programa computacional criado para obtenção do valor econômico das características analisadas. (Sequence of activities performed by the computer program designed to obtain the economic value of the features discussed).

No caso específico da PP14, foi desenvolvida uma analise em separado devido ao fato de que a metodologia que aqui foi apresentada estipula um valor fixo para o descarte de vacas de $20 \%$. Este fato implica em dizer que haverá um limite para a reposição dessas matrizes pelas novilhas que são geradas no sistema produtivo, com isso não se tem a maximização dessa característica.

Para corrigir tal problema, retirou-se o efeito da taxa de descarte de vacas, permitindo assim que toda novilha prenhe aos 14 meses seja utilizada como matriz. Devido a esse fato a quantidade de matrizes descartadas será exatamente igual a soma das quantidades de novilhas prenhes aos 14 e 24 meses. Com isso a uma inversão de raciocínio, onde o descarte de matrizes passa a ser em função da entrada de novas fêmeas e não da saída das fêmeas já consideradas matrizes.

Outra característica sob análise diferenciada é a habilidade de permanência, que basicamente está ligada à vida produtiva da matriz na propriedade.

Esta metodologia consiste, em termos de avaliação genética, classificar as matrizes no seguinte contexto, aquelas que apresentarem em 6 anos de vida 4 crias recebem o valor 1, e aquelas que estiverem fora disso, no caso para pior, receberá o valor 0 , com isso verifica-se que se trata de uma característica categórica, ou seja, ou ela é 1 ou 0.

Com isso, pressupõe-se que a um ganho genético na característica acarretará em um aumento na taxa de fertilidade do rebanho, e em conseqüência em uma diminuição na 


\section{ÍNDICE DE SELEÇÃO EM GADO CORTE SOB O ENFOQUE BIOECONÔMICO}

taxa de descarte das matrizes, considerando assim que o rebanho se tornará mais longevo. Para tanto em termos de estudos para determinar seu valor econômico, foi idealizado a seguinte equação:

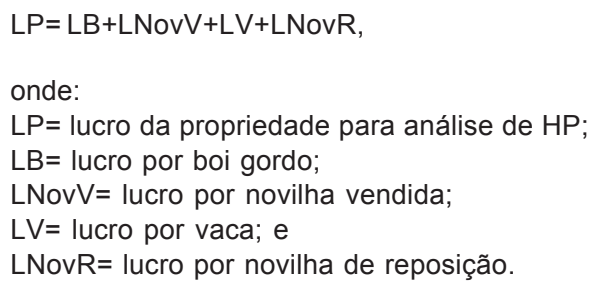

As equações de lucro são:

$\mathrm{LB}=\mathrm{N}^{*}\left(\left(\mathrm{NPV}^{*}\left(P A^{*} \operatorname{Rend}^{*} \mathrm{R} \$ / \mathrm{kg}\right)\right)-\left(\mathrm{NPV}{ }^{*} \mathrm{CB}\right)\right.$,

onde:

$\mathrm{N}=$ número de vacas em reprodução;

$\mathrm{NPV}=$ número de produtos por vaca;

$\mathrm{PA}=$ peso ao abate $(\mathrm{kg})$;

Rend= rendimento de carcaça (\%);

$\mathrm{R} \$ / \mathrm{kg}=$ preço por quilo vivo de boi gordo $(\mathrm{R} \$)$;

$\mathrm{CB}=$ custo por cabeça de boi gordo $(\mathrm{R} \$)$.

$L$ NovV $=N^{*}\left(\left(N P V^{*}\left(P A^{*} \operatorname{Rend}^{*} R \$ / k g\right)\right)-\left(N P V^{*} C N\right)\right.$,

onde:

$\mathrm{N}=$ número de vacas em reprodução;

$\mathrm{NPV}=$ número de produtos por vaca;

$\mathrm{PA}=$ peso ao abate $(\mathrm{kg})$;

Rend $=$ rendimento de carcaça (\%);

$\mathrm{R} \$ / \mathrm{kg}=$ preço por quilo vivo de novilha $(\mathrm{R} \$)$;

$\mathrm{CB}=$ custo por cabeça de novilha $(\mathrm{R} \$)$.

$L V=N^{*}\left(\left(V \operatorname{Desc}^{*}\left(P A^{*} \operatorname{Rend}^{*} R \$ / k g\right)\right)-C V\right.$,

onde:

$\mathrm{N}=$ número de vacas em reprodução;

VDesc $=$ percentual de vacas descartadas;

$\mathrm{PA}=$ peso ao abate $(\mathrm{kg})$;

Rend= rendimento de carcaça (\%);

$\mathrm{R} \$ / \mathrm{kg}=$ preço por quilo vivo de vaca gorda $(R \$)$; $C B=$ custo por cabeça de vaca gorda $(R \$)$.

LNovR $=\mathrm{N}^{*}\left(\mathrm{CCN}^{*} \%\right.$ NovR $)$,

onde:

$\mathrm{N}=$ número de vacas em reprodução;

$\mathrm{CCN}=$ custo de compra de novilhas;

$\%$ NovR= porcentagem de novilhas de reposição.
As variáveis de fertilidade, reposição de novilhas e descartes de vacas norteiam os cálculos, os dados de custo das categorias são obtidos através da soma dos custos diretos destas, onde compõe o custo de manutenção de boi, a soma dos custos diretos com bezerros, garrotes e bois; os custos de manutenção de novilhas, a soma dos custos diretos com bezerras, novilhas de 14 e 24 meses de idade; o custo de manutenção de vacas é composto pela soma dos custos diretos com vacas adultas e matrizes descartes.

Uma vez obtido o lucro do sistema, ocorre à oscilação percentual para mais na fertilidade e para menos na taxa de descarte de matrizes. A soma dos resultados divididos pela quantidade de matrizes no sistema gera o valor econômico da característica.

Para poder se comparar os diversos resultados obtidos para cada uma das características faz-se necessário a padronização dos resultados utilizando-se para isso o valor do desvio padrão genético ( a) para as características analisadas, sendo consideradas as seguintes estimativas: HP de $3,77 \%$; PP14 de 10,89\%; GPD245 de 6,66 kg; PD de $9,33 \mathrm{~kg}$ e PS de $13,97 \mathrm{~kg}$. Estes valores foram obtidos a partir das variâncias genéticas aditivas, observadas para as diferentes características, realizadas previamente a edição do sumário de touros Nelore CFM 2006 (sumário de touros Nelore CFM, 2006).

Com isso, o valor econômico obtido para cada característica deve ser dividido pelo seu respectivo desvio padrão genético para que se anule o efeito da unidade.

\section{RESULTADOSEDISCUSSÃO}

Uma vez definido o cenário padrão, foi possível realizar a simulação utilizando os recursos desenvolvidos para a obtenção dos valores econômicos das características de interesse zootécnico.

Com isso os resultados obtidos são apresentados na tabela II, onde todos os valores são gerados oscilando a caracterís- 
Tabela II. Valor econômico das características analisadas pelo modelo bioeconômico proposto. (Economic value of the characteristics analyzed by the proposed bio-economic model).

\begin{tabular}{|c|c|}
\hline Característica & $\begin{array}{c}\text { Valor } \\
\text { econômico R\$ }\end{array}$ \\
\hline Habilidade de permanência ano HP & 3,316 \\
\hline Precocidade sexual PP14 \% & 2,198 \\
\hline \multicolumn{2}{|l|}{ Fertilidade \% } \\
\hline novilhas 24 meses & 6,270 \\
\hline vacas $1^{\circ}$ cria & 1,062 \\
\hline vacas adultas & 14,751 \\
\hline \multicolumn{2}{|l|}{ Ganho de peso diário kg/dia } \\
\hline bezerros a desmama & 1,220 \\
\hline bezerras a desmama & 0,048 \\
\hline novilhas 14 meses & 0,556 \\
\hline novilhas 24 meses & 0,809 \\
\hline garrotes 18 meses & 1,775 \\
\hline bois 24 meses & 2,061 \\
\hline pós-desmama & 1,268 \\
\hline \multicolumn{2}{|l|}{ Peso kg } \\
\hline a desmama & 0,576 \\
\hline ao sobreano & 1,122 \\
\hline ao sobreano machos & 1,723 \\
\hline ao sobreano fêmeas & 0,519 \\
\hline \multicolumn{2}{|l|}{ Mortalidade \% } \\
\hline a desmama & 1,303 \\
\hline ao sobreano & 1,350 \\
\hline Rendimento de carcaça machos \% & 28,388 \\
\hline
\end{tabular}

tica analisada do seu valor médio para uma condição melhorada.

Analisando os valores obtidos chegase a uma escala de importância econômica, onde em primeiro lugar aparece rendimento de carcaça com $\mathrm{R} \$ 28,388 / \%$, seguido pelas características de fertilidade, que somadas chegam a $\mathrm{R} \$ 27,597 / \%$, as características de produção, peso e ganho de peso, que somadas atingem $\mathrm{R} \$ 11,677 / \mathrm{kg}$, e por fim as características de mortalidade com $\mathrm{R} \$$ $2,653 \%$.

Tais valores estão coerentes com os resultados obtidos por Brumatti (2002) e Formigoni (2002), que apesar de apresentarem valores menores, mantém a mesma classificação de importância, evidenciando que as questões monetárias ao longo do tempo não modificam a classificação de importância das características analisadas.

Em um estudo similar Jorge et al. (2006) desenvolveram um modelo bioeconômico para o cálculo do desempenho técnico e econômico de propriedades de ciclo completo e de cria em bovinos de corte, onde os resultados obtidos para a propriedade de ciclo completo foram de $\mathrm{R} \$ 0,40 / \mathrm{kg}$ para peso a desmama, $\mathrm{R} \$ 0,09 / \mathrm{kg}$ para peso da vaca adulta, $\mathrm{R} \$ 3,20 / 1 \%$ para taxa de prenhes e $\mathrm{R} \$ 10,15 / 1 \%$ para taxa de desmama, evidenciando a maior importância das características ligadas à fertilidade do rebanho quando comparadas às ligadas a produção.

Os mesmos autores, em um segundo trabalho, desenvolveram uma simulação para estudar a importância econômica das características zootécnicas em diferentes sistemas de produção de bovinos de corte, e verificaram que a taxa de desmama (TD) e o rendimento de carcaça (RC) foram as características de maior impacto econômico, apresentando valores que oscilaram de $\mathrm{R} \$ 3,51$ a $\mathrm{R} \$ 10,15 / \%$ de TD e de $\mathrm{R} \$ 23,89$ a $\mathrm{R} \$ 28,61 / \%$ de RC (Jorge et al., 2007).

Contudo essa comparação fica prejudicada uma vez que as características estão sob influência de suas unidades, onde não se permite comparar um resultado de fertilidade, que se expressa em percentagem, com um dado de peso, que se expressa em quilogramas por produto por período de análise.

Portanto, assumem-se cinco características para estudo dos valores econômicos: a habilidade de permanência (HP), a probabilidade de prenhes aos 14 meses (PP14), o ganho de peso pós desmama (GP245), o peso a desmama (PD) e o peso ao sobreano (PS). Tais características foram escolhidas por serem características economicamente relevantes, o que está de acordo com o que Bourdon (1998) e Ponzoni (1989), enfatizaram em seus estudos, ou seja, todas já são aplicadas em sistemas de avaliação genética no Brasil, conseqüen- 


\section{ÍNDICE DE SELEÇÃO EM GADO CORTE SOB O ENFOQUE BIOECONÔMICO}

temente com seus parâmetros genéticos conhecidos; e evidenciam as necessidades de uma propriedade quanto a características relacionadas à fertilidade, precocidade sexual e desempenho produtivo. A tabela III apresenta os valores genético-econômicos de cada característica sob análise.

Os valores genético-econômicos obtidos apresentam proporcionalidade similar no tocante ao maior peso as características de fertilidade do rebanho, aos resultados obtidos por Brumatti (2002), que encontrou valores iguais a 9,2 (HP); 11,13 (PP14); 6,39 (GPD245); 5,92 (PD); 11,95 (PS). Vale ressaltar que tal diferencial monetário se deve pelas oscilações econômicas entre cada período analisado e por diferenças metodológicas.

Tal proporcionalidade também se observa ao analisar os dados obtidos por Formigoni (2002), que encontrou valores de 10,82 (HP); 7,73 (PP14) e 10,82 (PD), evidenciando o maior peso das características vinculadas a fertilidade de rebanho, porém onde tais diferenças podem ser explicadas uma vez que o autor citado trabalhou com cenário de produção de cria e não com ciclo comple-

Tabela III. Valores genético-econômicos obtidos pelo modelo bioeconômico proposto. (Genetic-economic values obtained by the proposed bio-economic model).

\begin{tabular}{lccc}
\hline Características & VER $\$$ & $\sigma_{a}$ & VGE \\
\hline HP & 3,316 & 3,77 & 0,879 \\
PP14 & 2,198 & 10,89 & 0,201 \\
GPD245 & 1,268 & 6,66 & 0,190 \\
PD & 0,576 & 9,33 & 0,061 \\
PS & 1,122 & 13,97 & 0,080 \\
Total & - & - & 1,413
\end{tabular}

VE: valor econômico da característica; VGE: valor genético-econômico da característica; HP: habilidade de permanência; PP14: probabilidade de prenhes aos 14 meses; GPD245: ganho de peso pós-desmama; PD: peso a desmama; PS: peso ao sobreano. to, além de diferenças na metodologia de padronização dos dados.

Tal fato evidencia o que Phocas et al. (1998) alertaram em relação às diferenças encontradas, uma vez que os diversos trabalhos realizados na área diferem entre si em relação à metodologia desenvolvida, as circunstâncias de criação e produção, além de diferentes características analisadas, tornando-se difícil à comparação de resultados.

Entretanto, ressalta a importância das características reprodutivas, o que pode ser comprovado por Koots e Gibson (1998), que afirmaram serem as características fertilidade de vacas, mortalidade de bezerros, ingestão residual de alimentos e rendimento de carcaças, as principais características a serem trabalhadas em um programa de melhoramento genético animal.

Krupa et al. (2005) ao trabalharem com um modelo bioeconômico também concluíram que a vida produtiva da vaca, característica vinculada diretamente com a fertilidade do rebanho, foi a característica mais importante economicamente, seguida das características de peso a desmama e peso ao ano.

Tal importância das características de fertilidade também foi observada por Wolfova et al. (2005), ao concluírem que o desempenho reprodutivo e a longevidade das vacas são características de primordiais em todos os cenários estudados em seu trabalho.

Ao se analisar os valores genéticoeconômicos das características tornam-se evidentes que HP e PP14, estão claramente definidas quanto a seus objetivos, pois uma está diretamente relacionada à fertilidade e a outra à precocidade sexual, porém quanto às características produtivas optou-se em separá-las, com isso, ficaram agrupadas as características PD e GPD245, e se isolou o PS.

Vale ressaltar que as características, $\mathrm{PD}$ e PS, apresentam um alto valor de correlação, o que indica que o peso a desmama é um 


\section{BRUMATTI, FERRAZ, ELERE FORMIGONNI}

Tabela IV. Valores genético-econômicos e os valores das proporções entre as características reprodutivas e produtivas, obtidas pelo modelobioeconômico. (Genetic-economic values and the values of proportion between productive and reproductive traits, obtained by the model bio-economic).

\begin{tabular}{lc}
\hline Característica & VGE \\
\hline HP & 1,527 \\
PP14 & 0,350 \\
GPD245 & 0,331 \\
PD & 0,107 \\
PS & 0,139 \\
Proporção reprodução/produção & \\
$\quad$ (HP+PP14)/(GPD245+PD) & 4,28 \\
$\quad$ (HP+PP14)/(PS) & 13,46 \\
\hline
\end{tabular}

VGE: valor genético-econômico da característica; HP: habilidade de permanência; PP14: probabilidade de prenhes aos 14 meses; GPD245: ganho de peso pós-desmama; PD: peso a desmama; PS: peso ao sobreano.

componente do peso ao sobreano, fato que não se observa com a mesma intensidade entre o peso a desmama e o ganho de peso pós-desmama. Com isso, os valores encontrados são apresentados na tabela IV.

Os valores encontrados para a proporção entre as características reprodutivas sobre as produtivas, apresentam-se com a mesma tendência de outros autores, como pode ser visto nos valores apresentados por Phocas

\section{BIBLIOGRAFIA}

Agro-Pecuária-CFM. 2006. Sumário de touros Nelore CFM 2006. Agro-Pecuária-CFM. São José do Rio Preto. 56 pp.

Amer, P.R., Emmans, G.C. and Simm G. 2001. Breeding objectives for beef cattle in Ireland. Livest. Prod. Sci., 67: 223-239.

Barwick, S.A. 1994. B-OBJECT: a PC-Program to derive economic weights for beef cattle. In: Genetic Predition Workshop, 4. Proceedings. Beef ImprovementFederation. Kansas City. $10 \mathrm{pp}$.

Bittencourt, T.C.C. 2001. Estimativa de ponderadores econômicos para características de et al. (1998) e Barwick (1994), onde ambos encontraram o valor de 1,5 para a proporção, e aos valores encontrados por Newman et al. (1994), Nitter et al. (1994), Bittencourt (2001) e Macneil et al. (1994), que apresentam os seguintes valores 2,$0 ; 3,0$; 3,86 e 4,0 respectivamente.

\section{CONCLUSÕES}

Em termos de valores absolutos, a escala de importância das características apresentou-se da seguinte forma: rendimento de carcaça; características reprodutivas, as quais fertilidades, habilidade de permanência e precocidade sexual; características ponderais, como, ganhos de peso e pesos; e as características de mortalidade.

Em termos de valores genético-econômicos, utilizaram-se somente as características diretamente relacionadas à lucratividade do sistema, ou seja, as economicamente relevantes, e que possam ser utilizadas em programas de melhoramento genético animal no Brasil. Deste modo, os valores genético-econômicos obtidos para HP, PP14, GPD245, PD e PS foram, respectivamente iguais a 0,$879 ; 0,201 ; 0,190 ; 0,061 ; \mathrm{e}$ 0,080 .

A soma das características reprodutivas foi de 4,28 a 13,46 vezes mais importantes do que as características de crescimento, evidenciando as necessidades de se reformular os atuais índices de seleção.

importância econômica em gado de corte, usando equações de lucro. Tese (doutorado). Faculdade de Medicina de Ribeirão Preto. Universidade de São Paulo. Ribeirão Preto. 59 pp. Bourdon, R. and Golden, B. 2000. EPD's and economics: Determining the relative importance of traits. Disponível em: http://ansci.colostate. edu/ (10/10/2000).

Bourdon, R.M. 1998. Shortcomings of current genetic evaluation systems. J. Anim. Sci., 76: 2308.

Brumatti, R.C. 2002. Desenvolvimento de um modelo bioeconômico para a determinação de 


\section{ÍNDICE DE SELEÇÃO EM GADO CORTE SOB O ENFOQUE BIOECONÔMICO}

ponderadores econômicos utilizados em índices de seleção em gado de corte. Dissertação de Mestrado. Faculdade de Zootecnia e Engenharia de Alimentos. Universidade de São Paulo. Pirassununga. SP.

Formigoni, I.B. 2002. Estimação de valores econômicos para características componentes de índices de seleção em bovinos de corte. Dissertação de Mestrado. Faculdade de Zootecnia e Engenharia de Alimentos. Universidade de São Paulo. Pirassununga. SP.

Jorge, J., Cardoso, V.L. e Albuquerque, L.G. 2006. Modelo bioeconômico para cálculo de custos e receitas em sistemas de produção de gado de corte visando à obtenção de valores econômicos de características produtivas e reprodutivas. Rev. Bras. Zootecn., 35: 2187-2196.

Jorge, J., Cardoso, V.L. e Albuquerque, L.G. 2007. Objetivos de seleção e valores econômicos em sistemas de produção de gado de corte no Brasil. Rev. Bras. Zootecn., 36: 1549-1558.

Koots, K.R. and Gibson, J.P. 1998. Economic values for beef production traits from a herd level bioeconomic model. Can. J. Anim. Sci., 78: 29-45.

Krupa, E., Wolfova, M., Peskovicova, D., Huba, J. and Krupova, Z. 2005. Economic values of traits for Slovakian Pied cattle under different marketing strategies. Czech J. Anim. Sci., 50: 483-492.

Lôbo, R.B., Bezerra, L.A. e Oliveira, H.N. 1999. Avaliação genética de animais jovens, touros e matrizes. GMAC. Ribeirão Preto. 90 pp.

MacNeil, M.D., Newman, S., Enns, R.M. and StewartSmith, J. 1994. Relative economic values for
Canadian beef production using specialized sire and dam lines. Can. J. Anim. Sci., 74: 411417.

Newman, S., Macneil, M., Golden, B.L. and Barwick, S.A. 1994. Implementation and use os selection indexes in genetic evaluation schemes for beef cattle. In: Genetic Predition Workshop, 4. Proceedings. Beef Improvement Federation. Kansas City. 11 pp.

Nitter, G., Graser, H.U. and Barwick, S.A. 1994 Evaluation of advanced industry breeding schemes for Australian beef cattle. I. Method of evaluation and analysis for an example population structure. Aust. J. Agric. Res., 45: 1641-1656.

Paneto, J.C.C. 1998. Comparação entre as tendências genéticas e econômicas de um rebanho de suínos. Tese (Mestrado). Faculdade de Ciências Agrárias e Veterinárias, Universidade Estadual Paulista. Jaboticabal. 90 pp.

Phocas, F., Bloch, C., Chapelle, P., Bécherel, F., Renand, G. and Ménissier, F. 1998. Developing a breeding objective for a French purebred beef cattle selection programme. Livest. Prod. Sci., 57: 49-65.

Ponzoni, R.W. and Newman, S. 1989. Development breeding objectives for Australian beef cattle production. Anim. Prod., 49: 35-47.

Wolfova, M., Wolf, J., Zahradkova, R., Pribiyl, J., Dano, J., Krupa, E. and Kica, J. 2005. Breeding objectives for beef cattle used in different production system. 2. Model application to production system with the Charolais breed. Livest. Prod. Sci., 95: 217-230. 